\title{
Case Report: Pelvic Actinomycosis
}

Maxová K. ${ }^{1}$, Menzlová E. ${ }^{1}$, Kolařík D. ${ }^{2}$, Dundr P. $^{3}$, Halaška M. ${ }^{1}$

${ }^{1}$ Department of Obstetrics and Gynaecology, First Faculty of Medicine, Charles University in Prague and Hospital Bulovka, Prague, Czech Republic;

2Department of Gynaecology and Obstetrics, Third Faculty of Medicine, Charles University in Prague and Institute for the Care of Mother and Child, Prague, Czech Republic;

${ }^{3}$ Institute of Pathology, First Faculty of Medicine, Charles University in Prague and General University Hospital in Prague, Prague, Czech Republic

Received July 30, 2011; Accepted January 16, 2012.

Key words: Actinomycosis - Tumour - Antibiotics - Urinary bladder

Abstract: A case of pelvic actinomycosis is presented. The patient is 42-year-old female with a 5 weeks history of pelvic pain. An intrauterine device (IUD) was taken out 3 weeks ago. There is a lump length $9 \mathrm{~cm}$ between rectus muscles. Ultrasound, magnetic resonance imaging (MRI) and histology are used to make the diagnosis. Actinomycosis can mimic the tumour disease. The definitive diagnosis requires positive anaerobic culture or histological identification of actinomyces granulas. A long lasting antibiotic therapy is performed.

Mailing Address: Kateřina Maxová, MD., Department of Obstetrics and Gynaecology, First Faculty of Medicine, Charles University in Prague and Hospital Bulovka, Budínova 2, 18000 Prague 8, Czech Republic; Phone: +420 266083 236; e-mail: kacabamaxa@centrum.cz

(C) Charles University in Prague - Karolinum Press, Prague 2012 


\section{Introduction}

Actinomycosis is a chronic infectious disease caused by the microorganism of genera Actinomyces. Actinomyces is a part of normal mucosal flora in oropharynx, gastrointestinal tract (GIT) and female genital tract. It causes infection only when the surface of the tissue is broken, mainly due to the presence of foreign body (intrauterine device - IUD) or failure in integrity of mucosa. The infection spreads contiguously crossing the anatomic barriers. Distant lesions can be formed. Actinomycosis can be mistaken for malignant tumour in the pelvis. The definitive diagnosis requires positive anaerobic culture or histological identification of actinomyces granulas. Antibiotics for several weeks to months are required.

\section{Material and Methods}

A 42-year-old female was referred to our Department of Obstetrics and Gynaecology for 5 weeks lasting pain in the area below the navel. Her gynecologist thought of urinary tract infection therefore prescribed antibiotics. The treatment had no effect on pain. Her IUD was removed 3 weeks before this treatment that had been inserted before 20 months. It was already the second IUD multi load. The first one had been inserted for 40 months and was replaced by the second one without break. She negated fatigue, fever, loss of weight, nausea, dysuria and also gynecological discharge. The stool was regular. There was nothing important in her medical history.

Laboratory investigation: leucocytes 9.1, C-reactive protein (CRP) 6.4. Urine culture was sterile. An elongated lump was palpable between the rectus muscles below the navel and measured $9 \times 4 \mathrm{~cm}$. The lump was solid, fixed and painless. The ultrasound examination identified a non-homogenous infiltrate located in the

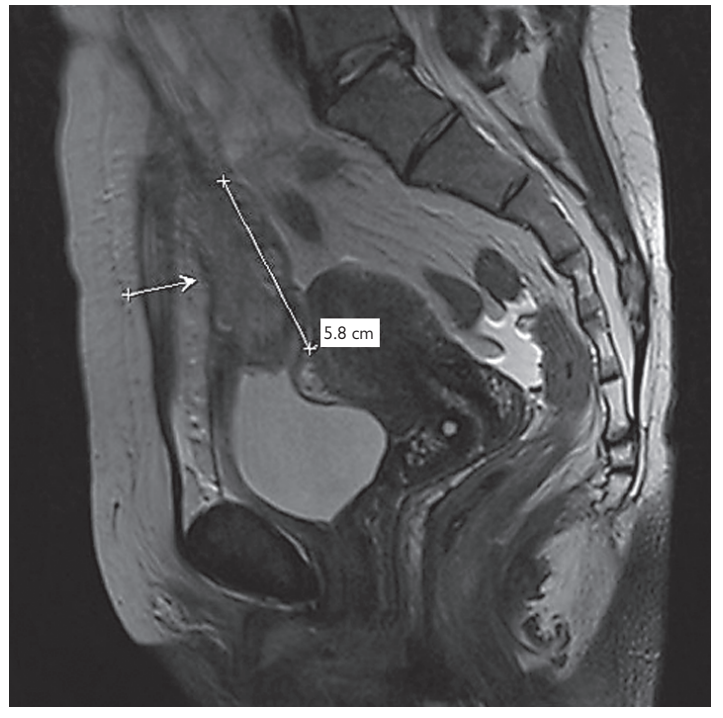

Figure 1 - Magnetic resonance imaging scan (arrow heads to the lump). 


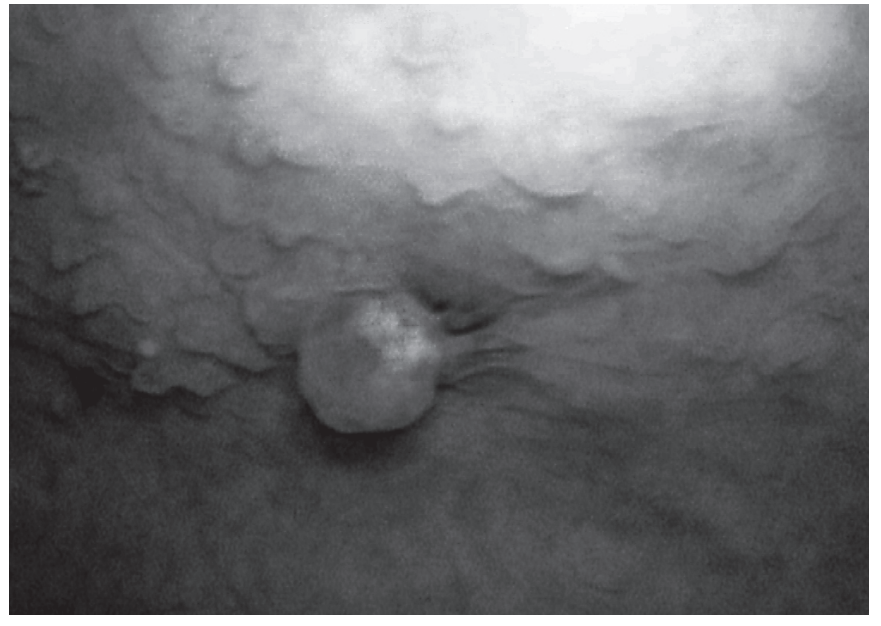

Figure 2 - Suspicious focus in the urinary bladder.

abdominal wall and the urinary bladder apex. The size was $92 \mathrm{~mm}$, thickness $48 \mathrm{~mm}$, width $72 \mathrm{~mm}$.

Magnetic resonance imaging (MRI) proved an oval lump size $58 \times 36 \times 35 \mathrm{~mm}$ which spread cranially between the rectus muscles toward the navel along the medial umbilical ligament. The lump infiltrated the adjacent peritoneum, lymphatic nodes weren't enlarged. The suspicion for tumorous process of the small pelvis was raised (Figure 1).

Cystoscopy was performed and a suspicious focus measuring $7 \times 7 \mathrm{~mm}$ was found in the vertex of the urinary bladder. There were yellowish substances at the top of the focus, thus, we gave a thought to actinomycosis. Samples for microscopic examination were taken (Figure 2 ).

The Tru-cut biopsy was performed and the diagnosis after histological examination of the actinomycosis was confirmed.

The patient was admitted to our Department of Obstetrics and Gynaecology for intensive parenteral antibiotic treatment. The type of antibiotics was consulted with reputable antibiotic centres. Penicillin G 20 million units was given for 21 days followed by Procaine Penicillin 3 million units daily.

After 3 days using the Procaine Penicillin the allergic reaction in the form of the maculopapulose exanthema appeared. We changed the antibiotics to peroral Duomox $1 \mathrm{~g}$ daily. The effect of therapy was controlled by ultrasound examination. The focus had a half of the previous size after 4 weeks of the treatment. After 12 weeks a quarter $(23 \times 15 \times 21 \mathrm{~mm})$.

The antibiotic administration continues without hospitalization and the treatment will last several months. Pelvic ultrasound scan is performed on monthly basis and liver enzymes are checked also monthly, cystoscopy is performed every two months. 


\section{Discussion}

Actinomycosis is a slowly progressing chronic infectious disease caused by Grampositive anaerobic bacteria genera Actinomyces, most frequently Actinomyces israelli. It is part of normal flora in oropharynx, GIT and female genital tract (Brown, 1973). If there is failure in integrity of mucosa microorganism can pass this barrier into the tissues and can form abscesses with surrounding granulation. The infection spreads contiguously crossing the anatomic barriers; hematogenous spread is less common (Makar et al., 1992). Nevertheless, distant lesions can be formed. The likelihood of colonization rises with increasing duration of IUD (Curtis and Pine, 1981).

The microorganisms multiply slowly creating microcolonies. They are surrounded by polymorphonuclear cells and are incrusted by calcium salts and sulphur granules formations not actually sulphur-containing but resembling such particles. These
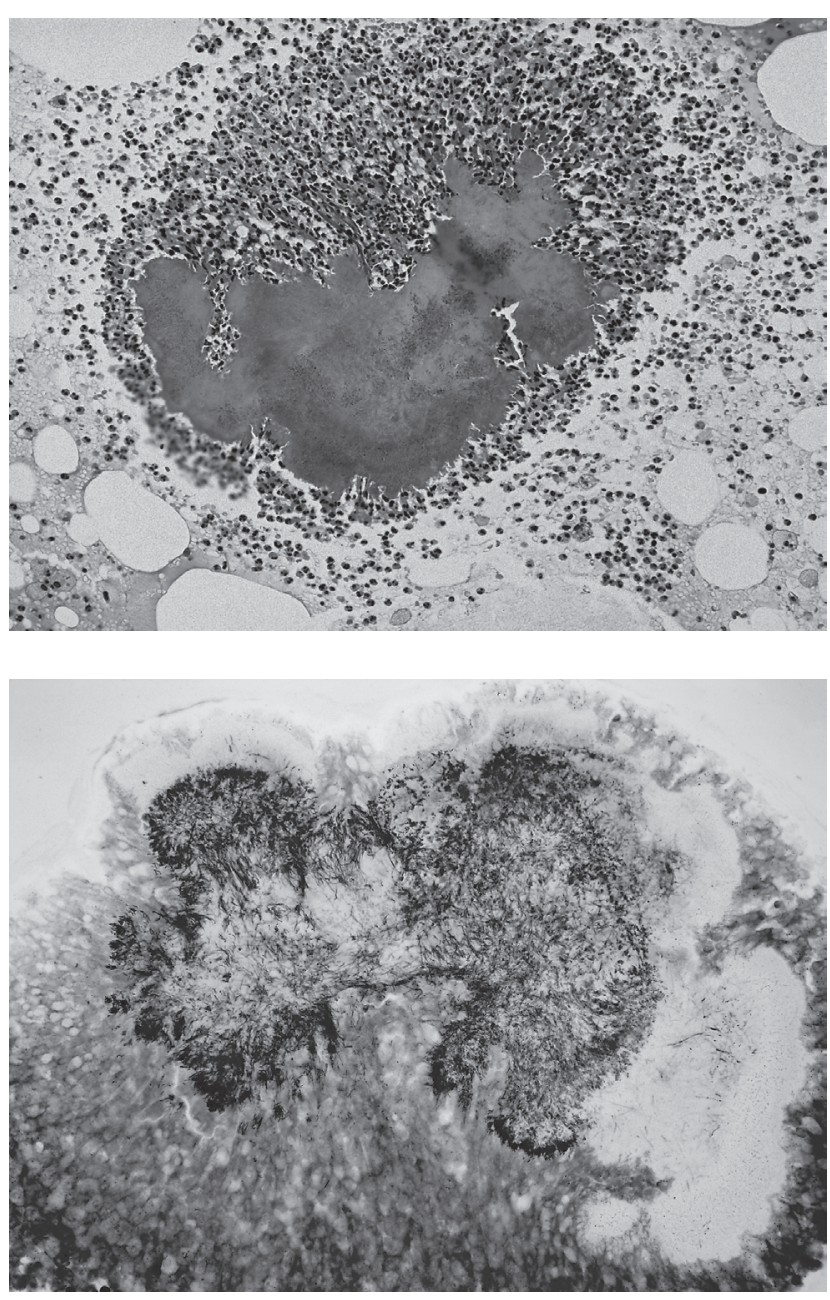

Figure 3 - Sulphur granules hematoxylin and eosin 250x.
Figure 4 - Sulphur granules Warthin-Starry 400x. 
granules contain progeny bacteria. The preliminary diagnosis can be made by examining these granules microscopically. The sulphur granules (up to $1 \mathrm{~mm}$ ) are macroscopically yellow/brown particles with a cauliflower appearance at low magnification; they are characteristic for actinomycosis. Gram staining reveals the typical beaded, branched, Gram-positive filamentous rods (Figures 3 and 4).

Based on the anatomic site of lesions four clinical forms are recognized: cervicofascial, thoracic, abdominal and pelvic. The microorganism causes the infection only when the tissue is broken; it requires the presence of foreign body (IUD) or failure in integrity of mucosa. The infection penetrates from perineum or abdominal wall into abdominal cavity and pelvic organs in pelvic or abdominal form (Koo et al., 2011). Actinomycosis can be then mistaken for malignant tumour. The definitive diagnosis requires positive anaerobic culture or histological identification of actinomyces granulas.

Standard causal therapy: antibiotics for several weeks to months. Benzylpenicillin is the first choice; in the case of resistance or complications second one is macrolids. Concerning dosage, initial treatment with parenteral benzylpenicillin 10-20 million units daily for 4-6 weeks can be followed by phenoxymetylpenicillin 2-4 grams for at least 6-12 months, or parenteral aminopenicillin $50 \mathrm{mg} / \mathrm{kg} /$ day for 4-6 weeks can be followed by peroral aminopenicillin $500 \mathrm{mg} / 8$ hours for 6 months. Dosage and treatment time depends on the scale of invasion, size of the lesion as well as on the compliance of the patient. Surgical intervention is limited to excision of abscesses and fistulas.

\section{Conclusion}

Pelvic actinomycosis can be mistaken with malignancy. We must think on this diagnosis especially in women using IUD.

\section{References}

Brown, J. R. (1973) Human actinomycosis. A study of 181 subjects. Hum. Pathol. 4, 319-330.

Curtis, E. M., Pine, L. (1981) Actinomyces in the vaginas of women with and without intrauterine contraceptive devices. Am. J. Obstet. Gynecol. 140, 880-884.

Koo, Y. J., Kwon, Y. S., Shim, J. U., Mok, J. E. (2011) Predictors associated with severity of pelvic actinomycosis. J. Obstet. Gynaecol. Res. 37(12), 1792-1796.

Makar, A. P., Michielsen, J. P., Boeckx, G. J., Van Marck, E. A. (1992) Primary actinomycosis of the urinary bladder. Br. J. Urol. 70, 205-206.

Maxová K.; Menzlová E.; Kolařík D.; Dundr P.; Halaška M. 